\title{
Molecular and Cellular Mechanisms of Lamina-specific Axon Targeting
}

\author{
Andrew D. Huberman ${ }^{1}$, Thomas R. Clandinin ${ }^{1}$, and Herwig Baier ${ }^{2}$ \\ ${ }^{1}$ Department of Neurobiology, Stanford University School of Medicine, Palo Alto, California 94305 \\ ${ }^{2}$ Department of Physiology, University of California, San Francisco, Programs in Neuroscience, \\ Genetics, and Developmental Biology, San Francisco, California 94158-2324 \\ Correspondence: herwig.baier@ucsf.edu
}

The specificity of synaptic connections is directly related to the functional integrity of neural circuits. Long-range axon guidance and topographic mapping mechanisms bring axons into spatial proximity of target cells and thus limit the number of potential synaptic partners. Synaptic specificity is then achieved by extracellular short-range guidance cues and cellsurface recognition cues. Neural activity may enhance the precision and strength of specific circuit connections. Here, we focus on one of the final steps of synaptic matchmaking: the targeting of synaptic layers and the mutual recognition of axons and dendrites within these layers.

$P^{2}$ erception and behavior are critically dependent on synaptic communication between specific neurons. Understanding how neurons achieve such "synaptic specificity" is therefore one of the most fundamental issues in developmental neuroscience. Langley's notion of "chemical relations" between synaptically connected neurons (Langley 1892) and Sperry's "chemoaffinity" hypothesis (Sperry 1963) provided a conceptual framework for the development of precise synaptic connections in the central nervous system. Sperry postulated that molecular interactions between neurons and their extracellular environment (including between and amongst axons and dendrites) ensure that connections form only between "appropriate" synaptic partners (Sperry 1963). This hypothesis has been confirmed by experimental work over the last four decades, most importantly by the identification of molecular cues that provide synaptic specificity (see Sanes and Yamagata 2009 for a recent comprehensive review). However, within this broad framework, a number of alternate mechanisms have been shown or proposed to play roles in specific aspects of such targeting processes. Here, we focus on mechanisms that underlie the formation of synaptic layers, a prominent anatomical feature of the visual system as well as many other areas of the CNS.

As reviewed previously (O'Leary 2010), the chemoaffinity principle underlies the developmental process of topographic mapping. Indeed, the precision with which neurons preserve the

Editors: Marc Tessier-Lavigne and Alex L. Kolodkin

Additional Perspectives on Neuronal Guidance available at www.cshperspectives.org

Copyright (C) 2010 Cold Spring Harbor Laboratory Press; all rights reserved; doi: 10.1101/cshperspect.a001743

Cite this article as Cold Spring Harb Perspect Biol 2010;2:a001743 
spatial relationships between the visual world and its representation in the brain is remarkable: Across animals ranging from flies to vertebrates, axons that bear signals from adjacent points in visual space invariably choose adjacent targets in the brain (Braitenberg 1967; Lemke and Reber 2005; Sperry 1963). Thus, position-dependent guidance of axons ensures that a visuotopic map develops. However, position in space is just one attribute of a visual stimulus; others include color, brightness, edge detection, and movement. If position in visual space is encoded by localized activation within a two-dimensional field of neurons, then these other features are encoded by local circuits that act both in series and in parallel and are reiterated many times across the field (Fig. 1). These local circuit modules are often envisioned as "columns" that lie orthogonal to the topographic map, with each column corresponding to a pixel in visual space and each level of the column representing a different, specific visual feature within that pixel, such as brightness, color, etc. (Fig. 1). How these columns acquire their laminated structure represents a developmental challenge of extraordinary scale. Although long-range axon guidance and topographic mapping no doubt contribute to restricting the astronomical number of potential synaptic partners, these mechanisms are clearly not sufficient; additional mechanisms must (and do) exist that act on a local scale to provide an additional level of positional information and cell-type-specific "chemoaffinity."

A prominent principle, which guides the formation of connections between specific cell types and is a characteristic feature of CNS architecture, is the concentration of synapses in small areas. These synapse clusters can take the form of planar layers or spherical glomeruli. Although glomeruli are a specialization that appears most prominent in the olfactory system, layers, or laminae, are an almost ubiquitous feature of central nervous system architecture. Indeed, even crude histological stains reveal that axons and dendrites often accumulate in neuropil (cell-body-free areas). Cell-type-specific or single-cell labeling has shown that, within individual neuropil layers, neurites and synapses are not distributed randomly. Rather, synaptic connections arising between neurons with the same or similar functional properties are localized to particular sublaminae that distinguish synapses with different properties (Fig. 1). The structural underpinnings of this functional principle are provided by mechanisms that ensure the lamina-specific branching of the corresponding neurites. How this enormous precision is achieved is the subject of intense investigations in the Drosophila, zebrafish, chick, and mouse visual systems. We will begin by describing three anatomical regions in these model organisms.
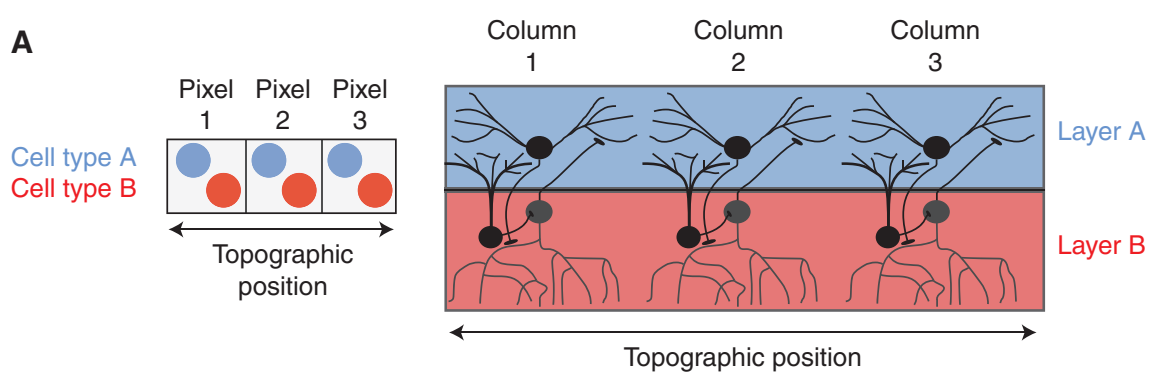

Figure 1. Laminae are a fundamental organizing unit of neural circuits. Each column corresponds to a single topographic position (e.g., location on the retina). Within each column, different cell types (shown type A: blue, and type B: red) respond to different features in the visual world, such as motion or luminance. These pixels are repeated many times over and thus cover all of visual space. A simple rule of "Cell type A connects to Layer A, etc." ensures that functional segregation is maintained in the connections from the retina to the target (parallel processing). Each pixel P1, P2, and P3 connects to a single column (C1, C2, and C3), establishing serial processing. Within each column, there are local circuits that, too, are layer-specific. Thus, laminae ensure functional specificity of both afferent-target connections and local circuit connections. 
Then, we will discuss three broad principles of layer-specific targeting in the visual system, namely cell-cell recognition, guidance by matrix cues, and activity-dependent sorting of axon terminals.

\section{LAMINAR ORGANIZATION OF THE VISUAL SYSTEM IN FLY, FISH, AND MOUSE}

Lamination and Sublamination in the Vertebrate Retina

The inner plexiform layer (IPL) of the vertebrate retina illustrates basic features of layered brain architecture. The IPL is sandwiched between two cell body layers, the inner nuclear layer (INL) and the ganglion-cell layer (GCL). In the retina, the synapses between bipolar cells, amacrine cells, and ganglion cells are found exclusively in the IPL. Their density is enormous: every cubic micrometer of the IPL contains $\sim 100$ synapses. Another set of retinal synapses, namely those formed between photoreceptors, horizontal cells, and bipolar cells are restricted to another very thin neuropil layer, the outer plexiform layer (OPL). This already precise targeting is made more complex by the fact that within each of these broad classes of cell types (amacrine, bipolar, and ganglion cells), there exist many subtypes. Indeed, there are between 50 and 120 morphologically distinguishable cell types in the vertebrate retina. The exact number depends on the species, with teleosts apparently holding the record (Badea and Nathans 2004; Connaughton et al. 2004; MacNeil et al. 1999; Mangrum et al. 2002; Masland 2001; Rockhill et al. 2002; Wagner and Wagner 1988). Each type has a characteristic (sub)laminar connection pattern in the OPL and/or IPL. Synapses between the 25-70 amacrine cell types, the 12 bipolar cell types, and the 15-20 ganglion cell types are stacked up within the IPL, forming at least eight anatomically separable sublaminae. These sublaminae are extremely thin: In the larval fish retina, they are just a few microns thick, barely accommodating a few synaptic terminals (Nevin et al. 2008). Frequently, but not always, dendrites and/or axons of retinal neurons are restricted to a single sublamina, giving rise to a single arbor with planar geometry. In fact, the vertical position(s) of branches within the neuropil layer, together with other morphological criteria, are frequently used to decide if two neurons belong to the same or different types. In the retinas of all vertebrates, there are many more cell types than anatomically distinguishable IPL sublaminae, arguing that a particular sublamina must necessarily be targeted by more than one cell type. Thus, there are at least three steps required to achieve synaptic specificity in the IPL: First neurites must be guided from the cell body into the neuropil; second, sublaminar targeting must occur; and, finally, selection of the synaptic partner amongst several cell types within each sublamina must take place. It is important to note that whereas bipolar cells and amacrine cells make layer-specific targeting choices strictly within the retina, ganglion cells make layer-specific choices for their dendrites in the retina, but also direct their axons out of the eye and into the visual brain areas, where they target particular neuropil layers, again in a type-specific fashion.

Layer-specific Photoreceptor Axon Targeting in Drosophila

The Drosophila visual system comprises the retina, and four optic ganglia, designated the lamina, the medulla, the lobula, and the lobula plate (reviewed in Meinertzhagen and Hanson 1993). As a compound eye, the retina is divided into an array of unit eyes, called ommatidia, each of which contains eight photoreceptors, $\mathrm{R}$ cells. Unlike vertebrate visual systems, in Drosophila, photoreceptor axons project directly into the brain, and display three distinct targeting preferences (Fig. 2A). Within each ommatidium, six R cells, designated R1-R6, extend axons into the outermost ganglion, the lamina, where they innervate a precise pattern of target cartridges such that each cartridge collects visual information from a single point in space, and neighboring cartridges are arranged to recreate a topographically correct representation of visual space (Braitenberg 1967; Kirschfeld 1967). These cartridges contain a reiterated 
A.D. Huberman, T.R. Clandinin, and H. Baier

A

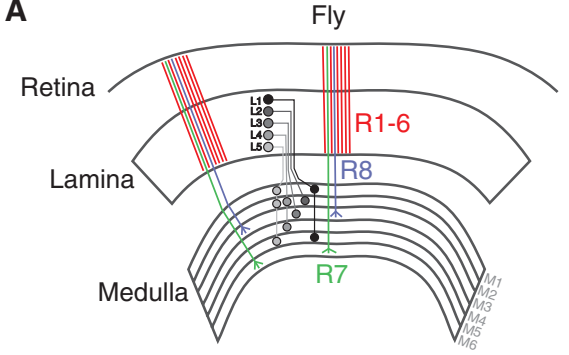

C

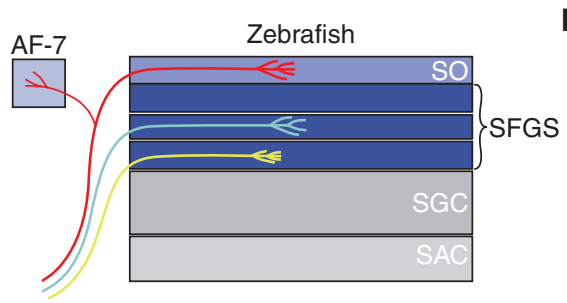

B

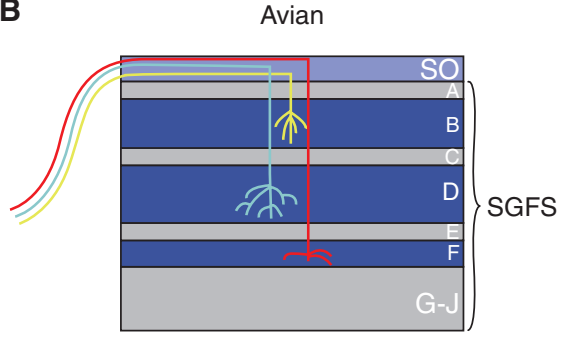

D

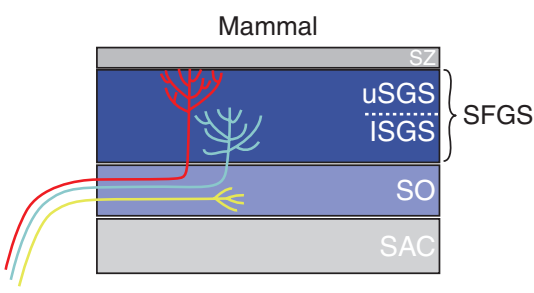

Figure 2. Lamina-specific retina-to-target connections across organisms. (A) Drosophila. Photoreceptors 1-6 (R1-6; red) in the retina project to their target in the brain, the lamina. Retinal photoreceptors R7 (green) and R8 (blue) project to different sublaminae of the medulla. Lamina neurons L1-L5 also send layer-specific connections to the medulla. (B) Chick. Axons from different types of RGCs (blue, yellow, and red) enter the tectum through the stratum opticum ( $\mathrm{SO}$ ) and then dive into one of three laminae (" $\mathrm{B}$, $\mathrm{D}$, or $\mathrm{F}$ " of the SGFS), where they establish synaptic connections onto neurons whose somas and/or dendrites reside in that specific layer. $(C)$ Zebrafish. Axons from different types of RGCs (blue, yellow, and red) enter the tectum from the rostral pole and directly target either the SO or one of the three sublaminae of the SFGS. Two deeper layers also receive sparse retinal input (not shown). The SO-projecting axons extend a collateral into the pretectal nucleus AF-7. (D) Mouse. Axons from different types of RGCs (blue, yellow, and red) all enter the tectum together through the SO. Some terminate in SO (yellow axons), whereas most turn dorsally to synapse either in the lower half (e.g., blue axon) or upper half (red axon) of the SGS (stratum griseum superficiale). The thin stratum zonale (SZ) receives sparse, if any RGC input.

array of projection neurons, designated L1-5 cells, which, in turn, project in an equally precise column and layer-specific fashion into the second optic ganglion, the medulla. Similarly, the axons of R7 and R8, the two remaining cells in each ommatidium, also innervate distinct layers within the medulla, within the same columns containing inputs from $\mathrm{L}$ cells that respond to visual signals from the same point in space. This targeting specificity emerges through a two-step process in which both R8 and R7 target precisely to a pair of temporary layers, before re-targeting to their final retinorecipient layers (Ting et al. 2005). Indeed, layerspecific targeting of many different neuron types is a broad organizational hallmark of the medulla, the lobula, and the lobula plate, and has been linked to specific behavioral functions in some cases (Bausenwein and Fischbach 1992;
Lee et al. 2001). Moreover, the retinotopic arrangement of columns that is established initially by photoreceptor axons innervating the lamina and medulla is preserved across all optic ganglia. Overall, this system is characterized by a remarkable degree of precision, with axons making precise projections throughout development, leading to the establishment of an essentially invariant adult connectivity pattern.

\section{Laminar Organization of the Vertebrate Retinotectal Projection}

Retinal ganglion cell axons, the outputs of the vertebrate retina, make both retinotopic and layer-specific targeting decisions within a variety of brain regions. Of these regions, perhaps the most intensively studied is the optic tectum (as 
it is known in fish and birds, also known as superior colliculus in mammals). Here, ganglion cell inputs innervate a specific subset of morphologically and molecularly distinct layers (Inoue and Sanes 1997; May 2006; Nevin et al. 2008). Thus, the well-studied retinotectal map is, in fact, a stack of several superimposed maps. Laminar segregation of retinal axons is found in all vertebrates, although the exact order of layers (and particularly the nomenclature) strikingly varies between birds, fish, and mammals (Fig. 2 B-D). It is clear that retinorecipient tectal layers are specialized to receive their inputs from specific subtypes of retinal ganglion cells (Huberman et al. 2008b; Huberman et al. 2009; Xiao et al. 2005; Yamagata and Sanes 1995), although the lamina-specific projection patterns of many additional ganglion cell types remain to be characterized.

In mice, at least some of these layer-specific targeting decisions emerge gradually during development, with a specific subtype of retinal ganglion cells, the tOFF-aRGCs, initially projecting broadly across the depth of the SC before retracting to a specific lamina. Within their specific layer of the colliculus, these laminated projections are further organized into a regular array of columns that likely reflect the precise array of ganglion cell bodies in the retina (Huberman et al. 2008b).

In zebrafish, laminar specificity of RGC projections is precise from the earliest developmental stages. RGCs project their axons into six target layers, called the stratum opticum (SO) at the surface of the tectum, three sublayers of the stratum fibrosum et griseum superficiale (SFGS), the stratum griseum centrale (SGC), and a deep layer bordering the stratum album centrale (SAC) and the stratum periventriculare (SPV) (Xiao et al. 2005). Each RGC sends out a single axon, which may form collaterals outside the tectum, but, as a rule, innervates just one tectal layer (Xiao and Baier 2007). The Pou4f3 (Brn3c)-expressing subset (which is composed of several types) projects into the SO and into the two deeper sublayers of the SFGS. A Pou4f3:mGFP-expressing transgenic reporter line (see below) has been used extensively to study laminar targeting of axons in the tectum, including in forward genetic screens (Xiao et al. 2005), and of RGC dendrites in the retina (Mumm et al. 2006).

\section{IN VIVO OBSERVATIONS OF LAYER FORMATION}

Watching the development of lamina-specific projections can often refine hypotheses regarding its cellular underpinnings. For instance, one can establish a timeline of events, as has been done for the zebrafish IPL, where it has been shown that amacrine neurites establish a sublaminated plexus several hours before RGC dendrites are growing in (Godinho et al. 2005; Kay et al. 2004; Mumm et al. 2006). Similar studies have illuminated the order of events surrounding the layer-specific targeting of photoreceptor and lamina neuron axons in the Drosophila medulla (Nern et al. 2008; Ting et al. 2005). However, it is important to emphasize that describing a sequence of events is no substitute for experiments that probe functional relationships between cells, molecules, and layer formation. For example, the mere observation of a diffuse projection that is sorted out over time, coincident with the onset of correlated neuronal activity, cannot be taken as evidence that the laminar segregation is dependent on patterned activity (Chalupa 2007). We will use the zebrafish IPL here as an example of the power of imaging of genetically labeled neurites as they innervate the correct sublamina to constrain developmental mechanism.

\section{Formation of the Zebrafish IPL}

Time-lapse imaging of fluorescently labeled amacrine cells and RGCs in the zebrafish retina has revealed the sequence of events that result in the formation of sublaminae (Fig. 3A-C). Here, amacrine cells are the first to extend neurites into the nascent IPL (Godinho et al. 2005). In fact, their processes form a band of neuropil in between their cell bodies, "pushing" one amacrine population into the future INL and another into the future GCL. The GCL-resident amacrine population will develop into the displaced amacrines of the mature 

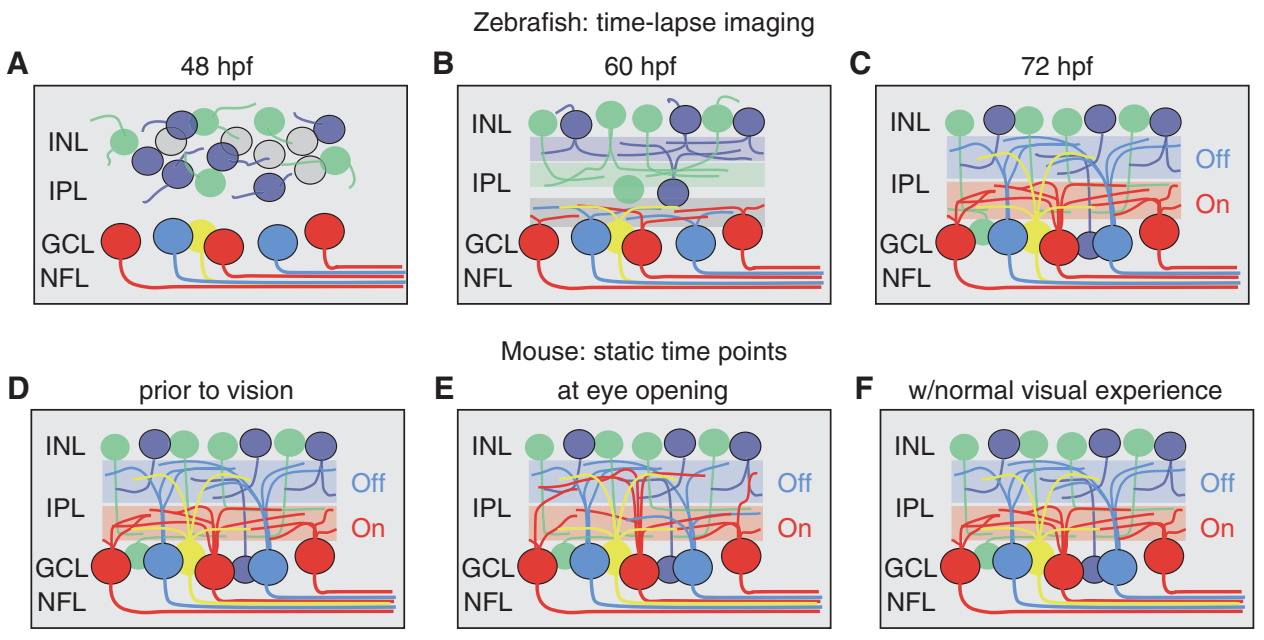

Figure 3. Developmental sequence leading to sublamination in the retinal inner plexiform layer. $(A-C)$ Summary of time-lapse imaging experiments in the developing zebrafish retina. (A) At 48 hours postfertilization (hpf), all amacrine cells (dark blue and green cells) are confined to inner nuclear layer (INL) and extend rudimentary processes in all directions. RGCs (red, light blue, and yellow) reside in the ganglion cell layer (GCL) and project their axons into the nerve fiber layer (NFL) but are yet to extend dendrites into the inner plexiform layer (IPL). (B) At $60 \mathrm{hpf}$, amacrine cells project their dendrites into separate strata (blue and green zones) in the IPL and RGCs extend dendrites into the IPL. Some amacrine somas begin to push through the IPL, toward the GCL; these are future "displaced amacrine cells." $(C)$ By $72 \mathrm{hpf}$, different types of RGCs (red, an On type, blue, an Off type, and yellow, On-Off type) confine their dendrites to separate strata in the proximal (On, red) or distal (Off, blue) half of the IPL. Displaced amacrine somas are present in the GCL. The dendrites of conventional and displaced amacrines and RGCs costratify in the correct sublaminae where they form synapses. $(D-F)$ Summary of static images of IPL stratification in mouse. Labeling conventions as in A-C. (D) Before vision, many RGCs target either the On or Off sublaminae of the IPL and cells that are destined to remain bistratified are bistratified at this stage (Huberman, unpubl. observations). (E) At eye opening ( $\sim 14$ days of age in mouse), many more bistratified RGCs exist than will persist into adulthood, although some bias in purely On- or purely Off-sublamina targeting is already present. $(F)$ With normal visual experience from eye opening, the IPL shows On-, Offand On-Off targeted RGC dendrites.

retina. Sublaminar organization emerges almost instantaneously once neurites have joined up (Godinho et al. 2005). Although RGCs are the first-born neurons in the retina and begin to differentiate earlier than amacrine cells (Kay et al. 2001; Kay et al. 2005; Masai et al. 2000), their dendrites first accumulate in the space below the future displaced amacrines and then move past them to contact the amacrine neurites. RGCs are not required for amacrine sublamination, although their absence destabilizes and slows down this process, as seen in RGC-deficient lakritz/atoh7 mutants (Kay et al. 2004).

Mumm et al. (2006) reported that most RGC dendrites directly target the pre-existing amacrine sublaminae (Fig. 3B,C). The four early-developing RGC dendritic strata are formed roughly in an inside-out fashion. First, the proximalmost sublamina appears, then the adjacent one, just distal to the first, etc. The fifth stratum then breaks the rule and forms between the second and the third sublamina. Importantly, individual RGCs do not adhere to this stereotyped sequence; some project to more distal sublaminae immediately. Also, laminar allegiances of RGC dendrites can change over time, with some branches being retracted from one layer and re-established in a different layer. In fact, the lamination strategies of single RGCs are highly diverse, and it is unclear if this reflects true cell-type diversity or some variability in their 
developmental trajectories (Mumm et al. 2006). Finally, bipolar axons arrive in the IPL after amacrine and RGC processes have formed connections (Schroeter et al. 2006).

These detailed observations led to the prediction of several molecular and cellular mechanisms. First, amacrine neurites are attracted to each other and carry type-specific cell-surface cues that ensure homotypic fasciculation and perhaps heterotypic repulsion, giving rise to sublaminae by cell-cell recognition. This interaction does not require the presence of their main postsynaptic partners, the RGCs. Second, RGC dendrites are attracted to the amacrine plexus or could be independently polarized to extend their dendrites preferentially into an apical direction toward the amacrine plexus. Third, RGC dendrites read surface cues provided by the preformed amacrine scaffold to correctly target sublaminae. Fourth, the ongoing reorganization of RGC dendrites indicates that the cell-cell recognition systems must be dynamically regulated. Finally, because this dynamic dendrite behavior extends well into mature stages (after $10 \mathrm{dpf}$ ) when fish larvae already use their visual sense to capture prey and avoid obstacles, these events must occur in the context of a functional visual system.

\section{LAMINA-SPECIFIC TARGETING BY CELL-CELL RECOGNITION}

In thinking about how layers might be specified during development by cell-surface cues, there are four broad categories of models to consider. First, and perhaps most intuitively, one could imagine that pre- and postsynaptic cells use some form of a combinatorial code of cellsurface molecules that directs their specific connectivity. In this view, pre- and postsynaptic cells express matching complements of ligands and receptors, so that only contacts between the correct cells are stabilized, whereas all others are disfavored (Fig. 4A). Such a mechanism would match pre- and postsynaptic cells, but does not provide an explicit mechanism for determining precisely where a given layer might form relative to neighboring layers. Second, one could imagine that there exists temporally dynamic expression of relatively ubiquitous celladhesion molecules that are switched on and off as axons are growing toward their target layers. In this view, once the axon reaches the appropriate layer, a generic adhesive factor is transiently engaged, terminating axon growth (Fig. $4 \mathrm{~B}, \mathrm{C})$. Third, one could imagine that one or more neurites in a layer could express attractive or repulsive axon guidance molecules, drawing appropriate axons either toward (or away) from their target layer. In this way, a target layer would act in a manner analogous to the wellstudied midline guidance pathways that have been described in both vertebrates and invertebrates (Dickson and Gilestro 2006). One could also imagine that quantitative differences in adhesive factors are used by incoming axons to sort into layers (Fig. 4D). Alternatively, gradients of adhesion or repellant cues could be expressed across the depth of single or several laminae. In both these views, pre- and postsynaptic cells might express "matching" levels of one or more adhesive factors, and by sequentially comparing relative levels of "stickiness" on each potential dendritic target, individual axons could optimize their target choice.

Of these four possible models, only the first three, combinatorial codes, complex temporal regulation of adhesion, and guidance cues, have as-yet been shown in vivo. Nonetheless, the extant data is grossly insufficient to account for all of the layer-specific targeting choices made in the brain, and undoubtedly new variations of these mechanisms are yet to be found.

\section{Specific Cell-surface Adhesion Molecules}

Perhaps the most intuitive model for how axons might choose their postsynaptic targets invokes the matched expression of homophilic or heterophilic cell-surface molecules on axons and dendrites that would allow axons to identify the correct postsynaptic partner from among a number of alternatives. This match could be at the level of a "unique" code in which only a single, homophilic molecule, or pair of heterophilic molecules, is matched between pre- and 
A.D. Huberman, T.R. Clandinin, and H. Baier

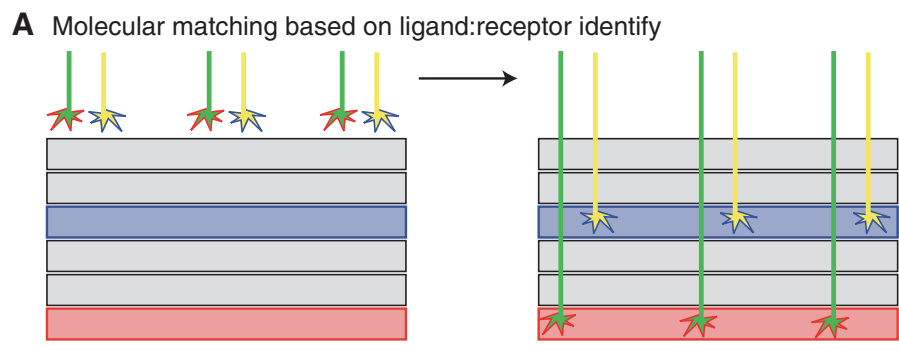

B Temporal coding 1: dynamic afferent expression

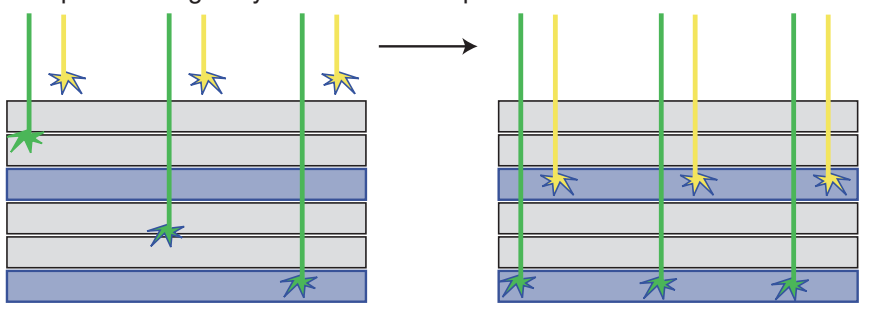

C Temporal coding 2: dynamic target expression

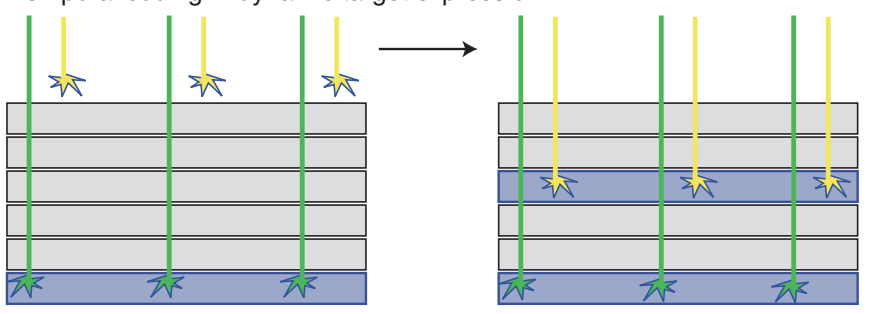

D Molecular matching based on ligand:receptor levels

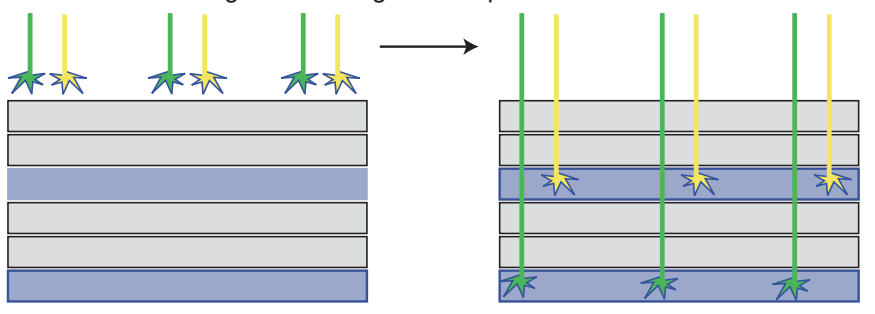

Figure 4. Molecular mechanisms of layer-specific axon targeting in the fly visual system. (A) Molecular matching of two different populations of photoreceptor axons (green or yellow) that each express different molecular recognition signals (red or blue). The target layers of these axons express either one recognition cue (blue) or a different molecular recognition cue (red), leading to a precise match between specific sets of axons and cells in specific laminae. (B) A temporal code could also underlie axon-target matching. One model is that a given set of axons (green) grow into the target and then turn on a surface recognition cue (blue), "locking" them into the most distal, bottom layer that expresses the matching cue. Later, a second group of axons (yellow) grow in to the target structure. They are already expressing the surface recognition cue (blue) and are directly targeting the first blue layer they encounter. $(C)$ An alternative temporal code model is that axons from different populations of neurons arrive already expressing the relevant layer-specific recognition cue. However, the expression of the matching cue is dynamic in the target. Thus, slight differences in timing of ingrowth will cause early arriving axons (here green) to synapse in certain layers, and other later arriving axons (yellow) to synapse in other layers with delayed expression of the cue. (D) Layer-specific axon targeting based on matching of axons and target layers expressing different levels of ligands and receptors. Here, the high-expressing growth cones (green axons) target the darker, high expressing (dark) blue target layers. The low expressing growth cones (yellow) target the also low expressing (lighter blue) target layers. 
postsynaptic cells, or could be defined through a combinatorial mechanism in which different combinations of cell-surface molecules need to be assessed in order to identify the appropriate match. This notion was first put forward in the context of the diverse layer-specific patterns of expression of cell-surface molecules observed in the chick tectum (Miskevich et al. 1998; Wohrn et al. 1998). Experimental support has predominantly come from studies of neurite patterning in the IPL, but fragmentary evidence in favor of this hypothesis has also emerged from studies of the fly visual system and the vertebrate tectum.

In the last few years, four closely related cell-surface adhesion molecules, all members of the immunoglobulin-domain-containing superfamily, have been identified as being important for sublaminar target recognition in the chick IPL (Yamagata and Sanes 2008; Yamagata et al. 2002). These molecules, called Sidekick (Sdk1), Sdk2, Dscam, and DscamL, are expressed in nonoverlapping subsets of bipolar cells, amacrine cells, and RGCs, and are each shared by cells that project to the same sublamina from either side of the IPL. All four molecules bind homophilically, as shown in vitro, and are concentrated at the sites of synaptic contacts in the IPL. Overexpressing Sdk1 (or Dscam) in cells that are normally negative for these factors results in misrouting of its processes to the Sdk1- (or Dscam-) rich sublamina. Conversely, removal of these molecules by gene knockdown perturbs the lamina-specific wiring. These results strongly suggest that these IgSF molecules (and related factors waiting to be discovered) are important elements of a cellsurface recognition code that ensures precise connectivity in the chick retina.

A recent study tested whether Dscam and Dscam-like 1 regulate synaptic and laminar specificity in mammals (Fuerst et al. 2009). The authors used anatomical methods to evaluate laminar-specific targeting of several different amacrine and RGC subtypes as well as more direct measurements of synaptic specificity such as electron microscopy and paired electrophysiological recordings. All of these data support the conclusion that in the absence of one or both
Dscams, retinal neuron recognize and form synapses with the appropriate partners. Especially remarkable is the fact that laminar and synaptic specificity still develops even though removal of Dscams causes retinal neurons to form dense aggregates with fasciculated dendrites (Fuerst et al. 2008, 2009). Thus, in mammals, Dscams regulate cell spacing but do not appear to mediate laminar-specific targeting or synaptic specificity as they do in the chick (Yamagata and Sanes 2008). The fact that appropriate synaptic partners still find one another in Dscam knockout animals suggests that adhesive cues do in fact regulate synaptic specificity in mammals. Still, the identity of adhesive cues that mediate overall target or cell-type-specific choices are unknown. Ango et al. (2004) established that an Ankryinrelated Ig molecule Neurofascin mediates subcellular targeting of interneuron synapses in the cortex-one of the few examples of an adhesion-based mechanism for synaptic specificity in mammals. Despite the elegance and simplicity of an adhesion-based code for synaptic specificity, repellants undoubtedly play an essential role as well. A recent study demonstrated that semaphorin initiated repulsion dictates synaptic specificity of a subset of spinal neurons by restricting their axons away from certain postsynaptic targets (Pecho-Vrieseling et al. 2009). Such results are especially noteworthy because, in addition to providing a primary example of a molecular determinant of synaptic specificity in the mammalian CNS, they suggest that laminar specificity may emerge through signals that drive axons away from inappropriate targets.

As it is relatively difficult to manipulate molecules in the chick, efforts in recent years have focused on developing the mouse as a model system to examine the mechanisms underlying specific axon targeting. Indeed, different subfamilies of IgSF molecules have been shown to be important for the pathfinding to olfactory glomeruli (Kaneko-Goto et al. 2008; Serizawa et al. 2006) and even the targeting of particular subcellular domains on Purkinje neuron dendrites in the cerebellum (Ango et al. 2004). In the visual system, evidence for the involvement of any of these IgSF molecules is scarce. More progress has been made in the 
colliculus than in the retina. In particular, the identification of markers for a number of subsets of retinal ganglion cells (Huberman et al. 2008b; Huberman et al. 2009; Kim et al. 2008; Siegert et al. 2009; Yonehara et al. 2008) has opened the possibility of determining the extent to which each of these ganglion cell subsets displays layer-specific targeting, and the mechanisms by which these targeting choices are made. Indeed, using one of these RGC cell types, the tOFF-aRGCs, as a model, it was shown that lamina-specific targeting of these axons can be achieved even in the absence of retinal activity waves (which play critical roles in organizing other aspects of targeting) (Huberman et al. 2008b). As these neurons appear to first innervate a broad region of the colliculus before becoming restricted to their retinorecipient layer, molecular cues must regulate pruning of the inappropriate initial connections of these cells.

In the fly visual system, the layer-specific targeting choices made by R7 and R8 photoreceptors have provided the most direct insight into the molecular underpinnings of a "codebased" targeting decision. In particular, the leucine-rich repeat protein Capricious appears to play a critical, specific role in matching $\mathrm{R} 8 \mathrm{ax}$ ons to their appropriate target layer. Capricious is expressed in both R8 axons (but not R7 axons), and their target layer, and mediates homophilic interactions in vitro (Shinza-Kameda et al. 2006). In capricious mutants, R8 axons mistarget, extending into the R7 layer. Strikingly, mis-expression of capricious in R7 axons is sufficient to redirect their targeting specifically to the layer of the medulla normally innervated by R8. Finally, the expression of capricious by R8 but not by R7 appears to be an integral part of the cell fate determination of these two cells-a transcription factor called Senseless that controls the expression of many R8-specific genes regulates the capricious promoter directly, and is in turn specifically repressed in R7 cells (Morey et al. 2008). Thus, the matched expression of capricious between R8 axons and their prospective targets appears to play an important, instructive role in the targeting of these cells.
Lamination in the Drosophila Visual System

by Dynamic Regulation of Cell Adhesion

In the fruit fly visual system, the molecular mechanisms underlying layer-specific targeting have been most closely examined in the context of the targeting choices made by R7, R8, and the L cells within the medulla. Strikingly, a number of lines of evidence show that a precisely timed pattern of expression of a relatively ubiquitous pair of cell-surface molecules, the classical cadherin, N-cadherin, and the receptor tyrosine phosphatase LAR appears to underlie the layerspecific targeting of a number of cell types. Both of these molecules were initially identified through studies of the developing Drosophila embryo, where embryos homozygous for mutations in either gene displayed a number of distinct defects in axon fasciculation, tract formation, guidance, and synapse formation (Iwai et al. 1997; Krueger et al. 1996). Given these broad, severe phenotypes, single cell somatic mosaic analysis of both genes in the visual system revealed the surprising result that R7 photoreceptors mutant for either of these genes displayed very specific defects in layer-specific targeting (Clandinin et al. 2001; Lee et al. 2003; Maurel-Zaffran et al. 2001). Further studies revealed that although mutations in these two genes have similar adult phenotypes, they play roles in distinct stages of the targeting process (Ting et al. 2005). Similar studies of $\mathrm{N}$-cadherin and LAR in L cells revealed that $\mathrm{N}$-cadherin plays both cell-autonomous and nonautonomous roles in directing the layerspecific branching patterns of specific cells (Nern et al. 2008). These latter studies also shown that changes in the fine-scale spatial and temporal pattern of $\mathrm{N}$-cadherin expression in specific neurites correlated well with the phenotypes seen in mutant cells (Nern et al. 2008).

Direct evidence in favor of the importance of the timing of $\mathrm{N}$-cadherin and LAR expression came through genetic studies of a transcription factor, Sequoia, which is expressed sequentially in R8 and R7 cells, and controls the timing of expression of both molecules (Petrovic and Hummel 2008) (Fig. 4B). In particular, sequoia mutant R7 and R8 cells target to inappropriate layers, while inducing prolonged expression of 
sequoia in $\mathrm{R} 8$ redirects these axons to the layer of the medulla normally innervated by R7. Strikingly, this effect of prolonging sequoia expression in R8 can be suppressed by removing $\mathrm{N}$-cadherin, arguing that $\mathrm{N}$-cadherin is a key effector. Together, these data argue that Sequoia controls the temporal pattern of $\mathrm{N}$-cadherin and LAR expression, and that this regulation is at least part of a mechanism that controls the competence of R7 and R8 axons to target to specific layers of the medulla. More broadly, these studies show that relatively ubiquitous cell-surface molecules with broad patterns of expression in the nervous system can be used to direct specific targeting decisions through precise timing of their expression on different cell surfaces.

Unfortunately, there is, as yet, no direct evidence that precisely timed patterns of expression of cell-adhesion molecules plays an instructive role in layer-specific targeting decisions made by axons in vertebrate visual systems. Nonetheless, it is intriguing to note that $\mathrm{N}$-cadherin plays a role in the development of layer-specific targeting of RGCs in the chick optic tectum. In particular, using a coculture system in which RGCs have simultaneous access to multiple tectal layers, a function-blocking antibody directed against $\mathrm{N}$-cadherin was sufficient to alter layer-specific targeting choices (Inoue and Sanes 1997). Moreover, mutations in zebrafish $\mathrm{N}$-cadherin affects lamination in the retina (Masai et al. 2003). Thus, dynamic regulation of such ubiquitous cell-adhesion molecules might be a broad theme of layerspecific targeting in a variety of experimental contexts.

\section{LAMINA-SPECIFIC GUIDANCE BY MATRIX CUES AND SECRETED FACTORS}

Although cell-cell recognition is important for synaptic specificity and might account for the "self-organization" of neurites into layers by selective aggregation, it is insufficient to explain the stereotypy of the order in which laminae and sublaminae are encountered in the brain. For this, we have to postulate either a temporal mechanism, which orders connections in space by a developmental sequence of ingrowth, or a laminar positional code, which is read out by, and is initially independent of, the ingrowing neurites. At least in the zebrafish visual system, which grows into adulthood, constantly adding new cells of all types and integrating them into pre-existing circuits, it is unlikely that a temporal sequence alone specifies the spatial order of layers. Rather, evidence is accumulating in this and other vertebrate species that the extracellular matrix is prepatterned to guide axons into their target layers and/or to restrict them from trespassing into a neighboring layer. These guidance cues could either attract subsets of axons into particular layers, or they could repel axons, thus preventing them from crossing into inappropriate territory. It is conceivable that their expression is in the form of bands, labeling unique vertical positions, or in the form of gradients, specifying a continuum of positions across the depth of the neuropil (Fig. 5). We review here only recent studies, omitting a large body of work on the role of extracellular matrix cues in axon guidance.

\section{Laminar Patterning by Basement Membrane-anchored Cues}

The notion that the basement membrane on the surface of the embryonic tectum could serve as a "laminar organizer" has received experimental support from studies of the zebrafish dragnet mutation, which causes a subset of RGC axons to stray outside of their normal layer (Xiao et al. 2005). The mutation causes disruptions in the structure of the basement membrane lining the tectal surface and was mapped to col4a5, a gene encoding the network-forming collagen IV $\alpha 5$ (Xiao and Baier 2007). Intriguingly, RGC axons in which Integrin $\beta 1$, an essential component of the main collagen IV receptor, was knocked down, showed normal targeting, suggesting that the axons are not responding directly to collagen IV. Rather, the guidance activity is likely due to one or more secreted factors that are bound to the basement membrane. Importantly, the effects of this mutation are phenocopied by enzymatic and genetic manipulations of heparan sulfate proteoglycans, 
A.D. Huberman, T.R. Clandinin, and H. Baier

A Zebrafish: directed growth
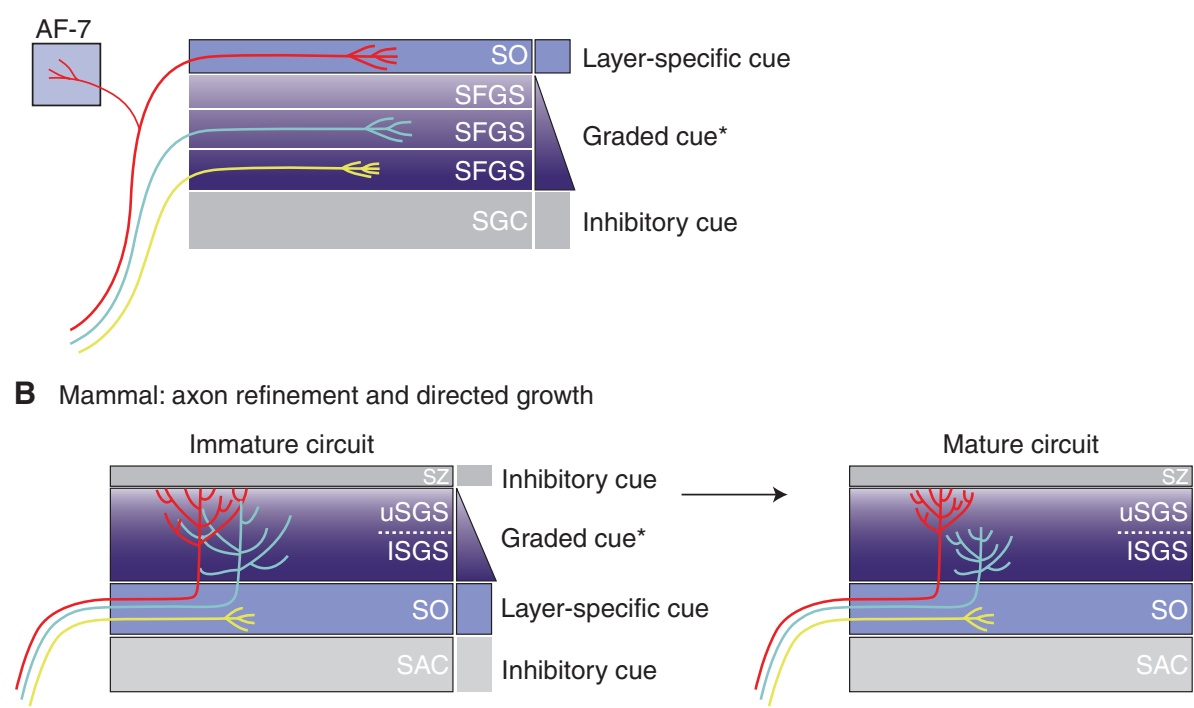

Figure 5. Established and putative molecular mechanisms for layer-specific axon targeting in the vertebrate tectum. (A) In zebrafish, RGC axons target the appropriate tectal laminae from the outset. This could be accomplished by guiding axons into their respective layers through repulsion and/or attraction. Guidance cues could be confined to narrow layers or expressed as gradients across the depth of the target (purple gradient from ventral to dorsal SFGC) and define domains of attraction and/or repulsion to subsets of RGC axons. (B) In mammals, some RGC axons initially target the wrong lamina and then refine to their correct target layer (light blue and red axons). Other RGC axons may target the correct lamina from the outset (yellow SO-targeting axon). The same targeting mechanisms presented in panel A could regulate these processes.

molecules that have been shown to act as cofactors for a variety of guidance molecules in other contexts. Thus, the basement membrane provides, directly, or indirectly, guidance cues to developing RGCs that are necessary to achieve appropriately restricted layer-specific targeting.

\section{Laminar Patterning by Extracellular Matrix Factors}

Studies in the optic tectum have revealed a number of cues, deposited in the extracellular matrix, that might help to organize layerspecific axonal targeting or other aspects of synaptic specificity. Among them are the large glycoproteins Tenascin-C, Tenascin-R, and Reelin, as well as the chondroitin sulfate proteoglycan Versican (Yamagata and Sanes 2005). Bands of Tenascin-R immunoreactivity border the retinorecipient layers, and Tenascin- $\mathrm{C}$ and $\mathrm{Te}-$ nascin- $\mathrm{R}$ have been shown in vitro to be repellent factors to RGC axons. These results suggest that the function of Tenascins may be to prevent axons from crossing into inappropriate territory (Becker et al. 2004; Perez and Halfter 1993). Loss of Reelin function in mouse and rat results in misrouting of axons that is restricted to the superficial retinorecipient layers of the tectum, where Reelin is expressed (Baba et al. 2007; Sakakibara et al. 2003). It is unclear if the observed defect is specific to laminar targeting, as this phenotype is obscured by disruptions of tissue architecture, which are caused by earlier functions of this secreted molecule in cell migration. However, a function for Reelin in circuit formation, as opposed to cell positioning, has been established in the retina, where AII amacrine cells express Dab1, an essential component of the Reelin signal transduction 
machinery. In both reeler and dab1 mutants, aberrant connections are formed in the IPL (Rice et al. 2001).

Most recently, the concept of extracellular laminar positional cues has been boosted by the identification of Nel, a glycoprotein containing five chordin-like and six epidermal growth factor-like domains (Jiang et al. 2009). In the developing chick tectum, expression of $\mathrm{Nel}$ is localized in specific laminae that retinal axons normally do not enter. Although the receptor is unknown, Nel-binding activity is detected on retinal axons both in vivo and in vitro. In vitro, Nel inhibits retinal axon outgrowth and induces growth cone collapse and axon retraction. These results suggest a role for Nel in the establishment of laminar specificity in the retinotectal projection.

\section{THE INFLUENCE OF NEURAL ACTIVITY ON TARGETING AND REFINEMENT OF LAMINA-SPECIFIC CONNECTIONS}

Neural activity is present in the developing vertebrate visual system long before the final pattern of synaptic connections is established, raising the possibility that it might drive, or modulate, laminar-specific targeting. In theory, this could occur if the neurons whose axons and/or dendrites are destined for particular layers were to show patterns of activity that are more similar to each other than to patterns associated with neurons that target to other layers. The retina shows spontaneous activity in the form of periodic, correlated firing of RGCs called retinal waves (Wong 1999). This correlated firing is important for topographic and eye-specific mapping of RGC axons in the LGN and SC (reviewed in Huberman et al. 2008a). However, it is important to note that neither topographic mapping nor eye-specific segregation reflect cell-type-specific targeting choices. Eye-specific "layers" are thus different from the functionally distinct laminar-specific targeting we've focused on thus far. Indeed, as mentioned above, if spontaneous neural activity is altered, tOff-alpha RGCs refine normally to their specific laminae in the LGN and SC, even though the same disruption in activity induces detectable changes in topographic and eye-specific refinement. Thus, true laminar recognition is independent of early retinal wave activity and may be independent of activity altogether. In the chick, the tectum can be excised and fixed with aldehydes, yet RGC axons still target the correct tectal laminae in such explants (Inoue and Sanes 1997). Moreover, a recent study in zebrafish showed that preventing synaptic vesicle release has no impact on layerspecific RGC axon targeting in the tectum (Nevin et al. 2008). Together, these studies provide strong evidence that laminar-specific axonal targeting is activity-independent. Whether activity can change those connections after they form seems unlikely, but nonetheless should be tested given that activity can desegregate eyespecific connections in the LGN after they form (Chapman 2000; Demas et al. 2006).

Is activity also dispensable for laminaspecific dendritic targeting in the IPL? Here, the evidence is mixed. In zebrafish, IPL sublaminae form even in the absence of synaptic transmission (Nevin et al. 2008). Similarly, selective suppression of On bipolar cell activity with $\mathrm{APB}$, creating a substantial imbalance of visual inputs to the RGCs, had no effect on their dendrite stratification, nor on the laminar targeting of amacrine or bipolar axons (Nevin et al. 2008). However, in kittens and ferrets, altering bipolar cell activity with the same drug prevents On- and Off-RGC dendrites from refining into specific sublaminae of the IPL (Bodnarenko and Chalupa 1993; Bodnarenko et al. 1995). Later studies in mouse generally arrived at the same conclusion; dark-rearing mice results in excessive numbers of On-Off bistratified RGCs (Tian and Copenhagen 2003). One possibility is that these different experimental outcomes simply reflect species-specific variations in wiring mechanisms. Still, a major problem in understanding the different results in fish versus mammals is that, even at maturity, mammalian retinas normally contain many On-Off bistratified RGCs, in addition to purely On or purely Off RGCs, and cell-type-specific markers have not yet been used in dark-rearing experiments. Thus, it is unclear if activity caused RGC destined to project to the ON sublamina 
to remain bistratified. A very recent study indicates that bipolar activity may not be required for layer-specific IPL targeting in mammals. Synaptic silencing of bipolar cells with tetanus toxin did not prevent RGCs from targeting the correct IPL sublamina (Kerschensteiner et al. 2009), similar to the results in fish (Nevin et al. 2008). Why then does dark rearing change the proportion of bistratified RGCs? One possibility is that early on, RGC dendrites are correctly targeted through yet to be identified (but clearly activity-independent) molecular mechanisms and then, once those connections form, activity is needed to maintain their laminar specificity (Fig. 3D-E). Only by the use of RGC subtype-specific markers can this be resolved. Fortunately, such markers now exist and these ideas can be directly tested.

\section{CONCLUSIONS}

To us, synaptic laminae represent both a fundamental anatomical unit in the brain as well as an organizing principle for neural development. Although a logically complete set of layer-specific targeting mechanisms is straightforward to define, only a subset of these mechanisms have been identified in vivo, and our molecular and cell biological understanding remains incomplete. However, new genetic tools and advancing imaging approaches are giving us an unprecedented capacity to unravel this critical step in synaptic specificity, and we anticipate that the coming years will bring many new insights.

\section{REFERENCES}

Ango F, di Cristo G, Higashiyama H, Bennett V, Wu P, Huang ZJ. 2004. Ankyrin-based subcellular gradient of neurofascin, an immunoglobulin family protein, directs GABAergic innervation at purkinje axon initial segment. Cell 119: 257-272.

Baba K, Sakakibara S, Setsu T, Terashima T. 2007. The superficial layers of the superior colliculus are cytoarchitectually and myeloarchitectually disorganized in the reelin-deficient mouse, reeler. Brain Res 1140: 205215.

Badea TC, Nathans J. 2004. Quantitative analysis of neuronal morphologies in the mouse retina visualized by using a genetically directed reporter. J Comp Neurol 480: $331-351$.
Bausenwein B, Fischbach KF. 1992. Activity labeling patterns in the medulla of Drosophila melanogaster caused by motion stimuli. Cell Tissue Res 270: 25-35.

Becker CG, Schweitzer J, Feldner J, Schachner M, Becker T. 2004. Tenascin-R as a repellent guidance molecule for newly growing and regenerating optic axons in adult zebrafish. Mol Cell Neurosci 26: 376-389.

Betley JN, Wright CV, Kawaguchi Y, Erdélyi F, Szabó G, Jessell TM, Kaltschmidt JA. 2009. Stringent specificity in the construction of a GABAergic presynaptic inhibitory circuit. Cell 139: 161-174.

Bodnarenko SR, Chalupa LM. 1993. Stratification of ON and OFF ganglion cell dendrites depends on glutamatemediated afferent activity in the developing retina. Nature 364: 144-146.

Bodnarenko SR, Jeyarasasingam G, Chalupa LM. 1995. Development and regulation of dendritic stratification in retinal ganglion cells by glutamate-mediated afferent activity. J Neurosci 15: 7037-7045.

Braitenberg V. 1967. Patterns of projection in the visual system of the fly. I. Retina-lamina projections. Exp Brain Res 3: 271-298.

Chalupa LM. 2007. A reassessment of the role of activity in the formation of eye-specific retinogeniculate projections. Brain Res Rev 55: 228-236.

Chapman B. 2000. Necessity for afferent activity to maintain eye-specific segregation in ferret lateral geniculate nucleus. Science 287: 2479-2482.

Clandinin TR, Lee CH, Herman T, Lee RC, Yang AY, Ovasapyan S, Zipursky SL. 2001. Drosophila LAR regulates R1-R6 and R7 target specificity in the visual system. Neuron 32: 237-248.

Connaughton VP, Graham D, Nelson R. 2004. Identification and morphological classification of horizontal, bipolar, and amacrine cells within the zebrafish retina. J Comp Neurol 477: 371-385.

Demas J, Sagdullaev BT, Green E, Jaubert-Miazza L, McCall MA, Gregg RG, Wong RO, Guido W. 2006. Failure to maintain eye-specific segregation in nob, a mutant with abnormally patterned retinal activity. Neuron 50: 247259.

Dickson BJ, Gilestro GF. 2006. Regulation of commissural axon pathfinding by slit and its Robo receptors. Annu Rev Cell Dev Biol 22: 651-675.

Fuerst PG, Koizumi A, Masland RH, Burgess RW. 2008. Neurite arborization and mosaic spacing in the mouse retina require Dscam. Nature 451: 470-474.

Fuerst PG, Bruce F, Tian M, Wei W, Elstrott J, Feller MB, Erskine L, Singer JH, Burgess RW. 2009. DSCAM and DSCAML1 function in self-avoidance in multiple cell types in the developing mouse retina. Neuron 64: 484497.

Godinho L, Mumm JS, Williams PR, Schroeter EH, Koerber A, Park SW, Leach SD, Wong RO. 2005. Targeting of amacrine cell neurites to appropriate synaptic laminae in the developing zebrafish retina. Development 132: 5069-5079.

Huberman AD, Feller MB, Chapman B. 2008a. Mechanisms underlying development of visual maps and receptive fields. Annu Rev Neurosci 31: 479-509. 
Huberman AD, Manu M, Koch SM, Susman MW, Lutz AB, Ullian EM, Baccus SA, Barres BA. 2008b. Architecture and activity-mediated refinement of axonal projections from a mosaic of genetically identified retinal ganglion cells. Neuron 59: 425-438.

Huberman AD, Wei W, Elstrott J, Stafford BK, Feller MB, Barres BA. 2009. Genetic identification of an On-Off direction-selective retinal ganglion cell subtype reveals a layer-specific subcortical map of posterior motion. Neuron 62: 327-334.

Inoue A, Sanes JR. 1997. Lamina-specific connectivity in the brain: regulation by $\mathrm{N}$-cadherin, neurotrophins, and glycoconjugates. Science 276: 1428-1431.

Iwai Y, Usui T, Hirano S, Steward R, Takeichi M, Uemura T. 1997. Axon patterning requires DN-cadherin, a novel neuronal adhesion receptor, in the Drosophila embryonic CNS. Neuron 19: 77-89.

Jiang Y, Obama H, Kuan SL, Nakamura R, Nakamoto C, Ouyang Z, Nakamoto M. 2009. In vitro guidance of retinal axons by a tectal lamina-specific glycoprotein Nel. Mol Cell Neurosci 41: 113-119.

Kaneko-Goto T, Yoshihara S, Miyazaki H, Yoshihara Y. 2008. BIG-2 mediates olfactory axon convergence to target glomeruli. Neuron 57: 834-846.

Kay JN, Link BA, Baier H. 2005. Staggered cell-intrinsic timing of ath5 expression underlies the wave of ganglion cell neurogenesis in the zebrafish retina. Development 132: 2573-2585.

Kay JN, Finger-Baier KC, Roeser T, Staub W, Baier H. 2001. Retinal ganglion cell genesis requires lakritz, a Zebrafish atonal Homolog. Neuron 30: 725-736.

Kay JN, Roeser T, Mumm JS, Godinho L, Mrejeru A, Wong RO, Baier H. 2004. Transient requirement for ganglion cells during assembly of retinal synaptic layers. Development 131: 1331-1342.

Kerschensteiner D, Morgan JL, Parker ED, Lewis RM, Wong RO. 2009. Neurotransmission selectively regulates synapse formation in parallel circuits in vivo. Nature 460: $1016-1020$.

Kim IJ, Zhang Y, Yamagata M, Meister M, Sanes JR. 2008. Molecular identification of a retinal cell type that responds to upward motion. Nature 452: 478-482.

Kirschfeld K. 1967. Die Projektion der optischen Umwelt auf das Raster der Rhabdomere im Komplexauge von Musca. Exp Brain Res 3: 248-270.

Krueger NX, Van Vactor D, Wan HI, Gelbart WM, Goodman CS, Saito H. 1996. The transmembrane tyrosine phosphatase DLAR controls motor axon guidance in Drosophila. Cell 84: 611-622.

Langley JN. 1892. On the origin from the spinal cord of the cervical and upper thoracic sympathetic fibres, with some observations on white and grey rami communications. Philos Trans R Soc Lond B Biol Sci 183: 85-124.

Lee RC, Clandinin TR, Lee CH, Chen PL, Meinertzhagen IA, Zipursky SL. 2003. The protocadherin Flamingo is required for axon target selection in the Drosophila visual system. Nat Neurosci 6: 557-563.

Lee CH, Herman T, Clandinin TR, Lee R, Zipursky SL. 2001 $\mathrm{N}$-cadherin regulates target specificity in the Drosophila visual system. Neuron 30: 437-450.
Lemke G, Reber M. 2005. Retinotectal mapping: new insights from molecular genetics. Annu Rev Cell Dev Biol 21: $551-580$.

MacNeil MA, Heussy JK, Dacheux RF, Raviola E, Masland RH. 1999. The shapes and numbers of amacrine cells: matching of photofilled with Golgi-stained cells in the rabbit retina and comparison with other mammalian species. J Comp Neurol 413: 305-326.

Mangrum WI, Dowling JE, Cohen ED. 2002. A morphological classification of ganglion cells in the zebrafish retina. Vis Neurosci 19: 767-779.

Masai I, Lele Z, Yamaguchi M, Komori A, Nakata A, Nishiwaki Y, Wada H, Tanaka H, Nojima Y, Hammerschmidt $\mathrm{M}$, et al. 2003. N-cadherin mediates retinal lamination, maintenance of forebrain compartments and patterning of retinal neurites. Development 130: 2479-2494.

Masai I, Stemple DL, Okamoto H, Wilson SW. 2000. Midline signals regulate retinal neurogenesis in zebrafish. Neuron 27: 251-263.

Masland RH. 2001. Neuronal diversity in the retina. Curr Opin Neurobiol 11: 431-436.

Maurel-Zaffran C, Suzuki T, Gahmon G, Treisman JE, Dickson BJ. 2001. Cell-autonomous and -nonautonomous functions of LAR in R7 photoreceptor axon targeting. Neuron 32: 225-235.

May PJ. 2006. The mammalian superior colliculus: laminar structure and connections. Prog Brain Res 151:321-378.

Meinertzhagen IA, Hanson TE. 1993. The development of the optic lobe. In The Development of Drosophila Melanogaster. M. B. a. A. Martinez-Arias. Cold Spring Harbor, NY, Cold Spring Harbor Press: 1363-1491.

Miskevich F, Zhu Y, Ranscht B, Sanes JR. 1998. Expression of multiple cadherins and catenins in the chick optic tectum. Mol Cell Neurosci 12: 240-255.

Morey M, Yee SK, Herman T, Nern A, Blanco E, Zipursky SL. 2008. Coordinate control of synaptic-layer specificity and rhodopsins in photoreceptor neurons. Nature 456: 795 799.

Mumm JS, Williams PR, Godinho L, Koerber A, Pittman AJ, Roeser T, Chien CB, Baier H, Wong RO. 2006. In vivo imaging reveals dendritic targeting of laminated afferents by zebrafish retinal ganglion cells. Neuron 52: 609-621.

Nern A, Zhu Y, Zipursky SL. 2008. Local N-cadherin interactions mediate distinct steps in the targeting of lamina neurons. Neuron 58: 34-41.

Nevin LM, Taylor MR, Baier H. 2008. Hardwiring of fine synaptic layers in the zebrafish visual pathway. Neural Dev 3: 36.

O'Leary DDM. 2010. Topographic mapping: The visual system. Cold Spring Harb Perspect Biol 2: a001768.

Pecho-Vrieseling E, Sigrist M, Yoshida Y, Jessell TM, Arber S. 2009. Specificity of sensory-motor connections encoded by Sema3e-Plxnd1 recognition. Nature 459: 842-846.

Perez RG, Halfter W. 1993. Tenascin in the developing chick visual system: distribution and potential role as a modulator of retinal axon growth. Dev Biol 156: 278-292.

Petrovic M, Hummel T. 2008. Temporal identity in axonal target layer recognition. Nature 456: 800-803.

Rice DS, Nusinowitz S, Azimi AM, Martinez A, Soriano E, Curran T. 2001. The reelin pathway modulates the 
A.D. Huberman, T.R. Clandinin, and H. Baier structure and function of retinal synaptic circuitry. Neuron 31: 929-941.

Rockhill RL, Daly FJ, MacNeil MA, Brown SP, Masland RH 2002. The diversity of ganglion cells in a mammalian retina. J Neurosci 22: 3831-3843.

Sakakibara S, Misaki K, Terashima T. 2003. Cytoarchitecture and fiber pattern of the superior colliculus are disrupted in the Shaking Rat Kawasaki. Brain Res Dev Brain Res 141: $1-13$.

Sanes JR, Yamagata M. 2009. Many paths to synaptic specificity. Annu Rev Cell Dev Biol 25: 8.1-8.25.

Schroeter EH, Wong RO, Gregg RG. 2006. In vivo development of retinal ON-bipolar cell axonal terminals visualized in nyx::MYFP transgenic zebrafish. Vis Neurosci 23: 833-843.

Serizawa S, Miyamichi K, Takeuchi H, Yamagishi Y, Suzuki M, Sakano H. 2006. A neuronal identity code for the odorant receptor-specific and activity-dependent axon sorting. Cell 127: 1057-1069.

Shinza-Kameda M, Takasu E, Sakurai K, Hayashi S, Nose A 2006. Regulation of layer-specific targeting by reciprocal expression of a cell adhesion molecule, capricious. Neuron 49: 205-213.

Siegert S, Scherf BG, Del Punta K, Didkovsky N, Heintz N, Roska B. 2009. Genetic address book for retinal cell types. Nat Neurosci 12: 1197-1204.

Sperry RW. 1963. Chemoaffinity in the orderly growth of nerve fiber patterns and connections. Proc Natl Acad Sci 50: 703-710.

Tian N, Copenhagen DR. 2003. Visual stimulation is required for refinement of ON and OFF pathways in postnatal retina. Neuron 39: 85-96.

Ting CY, Yonekura S, Chung P, Hsu SN, Robertson HM, Chiba A, Lee CH. 2005. Drosophila N-cadherin functions in the first stage of the two-stage layer-selection process of R7 photoreceptor afferents. Development 132: 953-963.
Wagner HJ, Wagner E. 1988. Amacrine cells in the retina of a teleost fish, the roach (Rutilus rutilus): a Golgi study on differentiation and layering. Philos Trans $R$ Soc Lond B Biol Sci 321: 263-324.

Wohrn JC, Puelles L, Nakagawa S, Takeichi M, Redies C. 1998. Cadherin expression in the retina and retinofugal pathways of the chicken embryo. J Comp Neurol 396: 20-38.

Wong RO. 1999. Retinal waves: stirring up a storm. Neuron 24: 493-495.

Xiao T, Baier H. 2007. Lamina-specific axonal projections in the zebrafish tectum require the type IV collagen Dragnet. Nat Neurosci 10: 1529-1537.

Xiao T, Roeser T, Staub W, Baier H. 2005. A GFP-based genetic screen reveals mutations that disrupt the architecture of the zebrafish retinotectal projection. Development 132: 2955-2967.

Yamagata M, Sanes JR. 1995. Target-independent diversification and target-specific projection of chemically defined retinal ganglion cell subsets. Development 121: 3763-3776.

Yamagata M, Sanes JR. 2005. Versican in the developing brain: lamina-specific expression in interneuronal subsets and role in presynaptic maturation. J Neurosci 25: 8457-8467.

Yamagata M, Sanes JR. 2008. Dscam and Sidekick proteins direct lamina-specific synaptic connections in vertebrate retina. Nature 451: 465-469.

Yamagata M, Weiner JA, Sanes JR. 2002. Sidekicks: synaptic adhesion molecules that promote lamina-specific connectivity in the retina. Cell 110: 649-660.

Yonehara K, Shintani T, Suzuki R, Sakuta H, Takeuchi Y, Nakamura-Yonehara K, Noda M. 2008. Expression of SPIG1 reveals development of a retinal ganglion cell subtype projecting to the medial terminal nucleus in the mouse. PLoS One 3: e1533. 


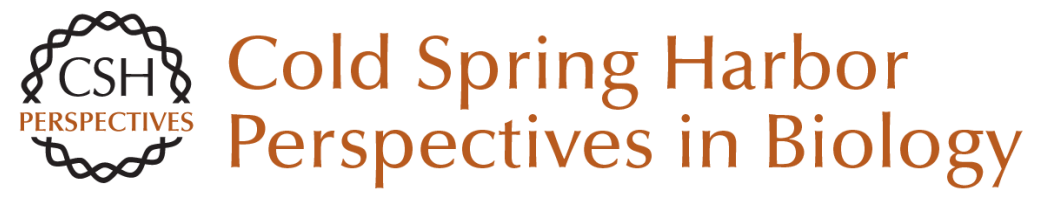

\section{Molecular and Cellular Mechanisms of Lamina-specific Axon Targeting}

Andrew D. Huberman, Thomas R. Clandinin and Herwig Baier

Cold Spring Harb Perspect Biol 2010; doi: 10.1101/cshperspect.a001743

Subject Collection Neuronal Guidance

Mechanisms and Molecules of Neuronal Wiring: A Primer

Alex L. Kolodkin and Marc Tessier-Lavigne

Guidance Molecules in Axon Pruning and Cell

Death

Pierre Vanderhaeghen and Hwai-Jong Cheng

Initiating and Growing an Axon

F. Polleux and William Snider

Navigating Intermediate Targets: The Nervous System Midline

Barry J. Dickson and Yimin Zou

Cellular Strategies of Axonal Pathfinding Jonathan Raper and Carol Mason

Guidance Molecules in Axon Regeneration Roman J. Giger, Edmund R. Hollis II and Mark H. Tuszynski

Signaling from Axon Guidance Receptors Greg J. Bashaw and Rüdiger Klein

Visual Map Development: Bidirectional Signaling, Bifunctional Guidance Molecules, and Competition

David A. Feldheim and Dennis D. M. O'Leary
Wiring the Brain: The Biology of Neuronal Guidance Alain Chédotal and Linda J. Richards

Guidance Molecules in Synapse Formation and Plasticity Kang Shen and Christopher W. Cowan

The Growth Cone Cytoskeleton in Axon Outgrowth and Guidance Erik W. Dent, Stephanie L. Gupton and Frank B. Gertler

Topographic Mapping--The Olfactory System Takeshi Imai, Hitoshi Sakano and Leslie B. Vosshall

Self-avoidance and Tiling: Mechanisms of

Dendrite and Axon Spacing Wesley B. Grueber and Alvaro Sagasti

Trafficking Guidance Receptors Bettina Winckler and Ira Mellman

Axon Guidance Molecules in Vascular Patterning Ralf $H$. Adams and Anne Eichmann

Human Genetic Disorders of Axon Guidance Elizabeth C. Engle

For additional articles in this collection, see http://cshperspectives.cshlp.org/cgi/collection/

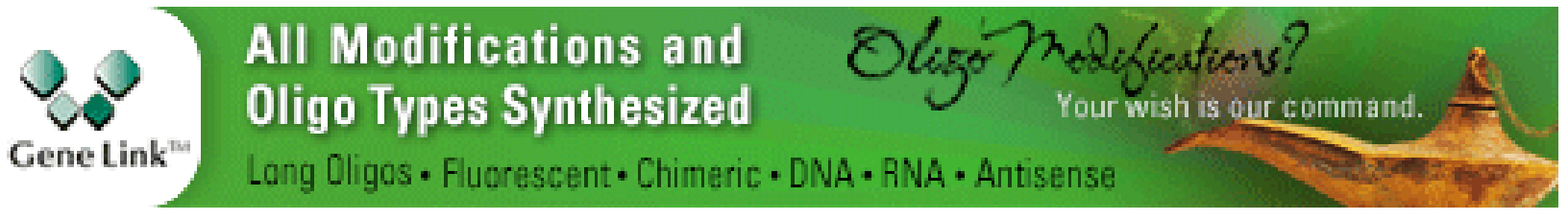

Copyright @ 2010 Cold Spring Harbor Laboratory Press; all rights reserved 